\title{
WISE-2005: effect of aerobic and resistive exercises on orthostatic tolerance during 60 days bed rest in women
}

\author{
Patrick Guinet • Suzanne M. Schneider • Brandon R. Macias • \\ Donald E. Watenpaugh • Richard L. Hughson · Anne Pavy Le Traon • \\ Jean-Yves Bansard · Alan R. Hargens
}

Accepted: 2 February 2009 / Published online: 27 February 2009

(c) The Author(s) 2009. This article is published with open access at Springerlink.com

\begin{abstract}
Cardiovascular deconditioning after long duration spaceflight is especially challenging in women who have a lower orthostatic tolerance (OT) compared with men. We hypothesized that an exercise prescription, combining supine aerobic treadmill exercise in a lower body negative pressure (LBNP) chamber followed by $10 \mathrm{~min}$ of resting LBNP, three to four times a week, and flywheel
\end{abstract}

\section{P. Guinet}

Department of Anaesthesiology and Intensive Care,

University of Rennes, Rennes, France

\section{S. M. Schneider}

Department of Health, Exercise and Sports Sciences,

University of New Mexico, Albuquerque, NM, USA

\section{B. R. Macias · A. R. Hargens}

Department of Orthopaedic Surgery, UCSD Medical Center, University of California San Diego, San Diego, CA, USA

\section{E. Watenpaugh}

Department of Integrative Physiology,

University of North Texas Health Science Center Fort Worth, Texas, USA

\section{R. L. Hughson}

Faculty of Applied Health Sciences,

University of Waterloo, Waterloo, ON, Canada

\author{
A. P. Le Traon \\ Department of Physiology, University of Brest, Brest, France \\ J.-Y. Bansard \\ Laboratoire de Traitement du Signal et de l'Image, \\ INSERM U642, Université Rennes 1, Rennes, France \\ P. Guinet $(\square)$ \\ Anesthesiology and Intensive Care, Pontchaillou Hospital, \\ 2 rue Henri Le Guillou, 35000 Rennes, France \\ e-mail: patrick.guinet@chu-rennes.fr
}

resistive training every third day would maintain orthostatic tolerance (OT) in women during a 60-day head-down-tilt bed rest (HDBR). Sixteen women were assigned to two groups (exercise, control). Pre and post HDBR OT was assessed with a tilt/LBNP test until presyncope. OT time (mean $\pm \mathrm{SE}$ ) decreased from $17.5 \pm 1.0 \mathrm{~min}$ to $9.1 \pm$ $1.5 \mathrm{~min}(-50 \pm 6 \%)$ in control group $(P<0.001)$ and from $19.3 \pm 1.3 \mathrm{~min}$ to $13.0 \pm 1.9 \mathrm{~min}(-35 \pm 7 \%)$ in exercise group $(P<0.001)$, with no significant difference in OT time between the two groups after HDBR $(P=0.13)$. Nevertheless, compared with controls post HDBR, exercisers had a lower heart rate during supine rest (mean $\pm \mathrm{SE}, 71 \pm 3$ vs. $85 \pm 4, P<0.01)$, a slower increase in heart rate and a slower decrease in stroke volume over the course of tilt/ LBNP test $(P<0.05)$. Blood volume (mean \pm SE) decreased in controls $(-9 \pm 2 \%, P<0.01)$ but was maintained in exercisers $(-4 \pm 3 \%, P=0.17)$.

Our results suggest that the combined exercise countermeasure did not significantly improve OT but protected blood volume and cardiovascular response to sub tolerance levels of orthostatic stress.

Keywords Simulated microgravity - Cardiovascular deconditioning $\cdot$ Exercise countermeasure $\cdot$ Lower body negative pressure

\section{Introduction}

The transition from supine to standing posture requires complex physiological mechanisms to maintain cerebral blood flow. Exposure to actual or simulated microgravity by head-down-tilt bed rest (HDBR) alters these adaptive mechanisms and causes orthostatic intolerance as well as decreased exercise capacity (Convertino et al. 1989; Butler 
et al. 1991; Vernikos et al. 1993; Buckey et al. 1996; Pavy-Le Traon et al. 1999). Several factors contribute to orthostatic intolerance: moderate hypovolemia (Buckey et al. 1996; Custaud et al. 2002), decreased stroke volume (Buckey et al. 1996), myocardial atrophy (Levine et al. 1997), reduced baroreflex sensitivity (Sigaudo-Roussel et al. 2002) and increased distensibility of lower extremity blood vessels (Convertino et al. 1989; Belin de Chantemele et al. 2004b). Furthermore, females have a lower orthostatic tolerance than males (Convertino 1998; Fu et al. 2004). Lower orthostatic tolerance is observed after short-duration space flights (5-16 days) with a four times higher incidence of presyncopal symptoms during a $10 \mathrm{~min}$ head-up tilt test in female astronauts compared with males (28 and 7\%, respectively) (Harm et al. 2001). Nevertheless, data concerning women in actual or simulated microgravity remain sparse.

Several countermeasures based on the microgravityinduced changes described above have proven only partly effective to counteract orthostatic intolerance. Restoration of blood volume (9-14 days spaceflight) (Buckey et al. 1996), moderate levels of aerobic exercise (during 916 days spaceflight or a 15-day HDBR, respectively) (Lee et al. 1999; Schneider et al. 2002), or bouts of maximal exercise (90-day HDBR) (Belin de Chantemele et al. 2004a) are not able to maintain orthostatic tolerance at pre flight or pre HDBR levels. Periods of lower body negative pressure (LBNP) applied during HDBR provide beneficial effects on plasma volume, baroreflex sensitivity and lower limb vascular distensibility (Arbeille et al. 1995; Traon et al. 1995). Orthostatic tolerance is maintained by $1 \mathrm{~h}$, progressively increasing to $2 \mathrm{~h}$ of daily, low-level LBNP sessions (28 mmHg) during a 30-day HDBR (Guell et al. 1991). Nevertheless, such prolonged LBNP sessions devoted only to preservation of orthostatic tolerance, are incompatible with in-flight timelines.

The combination of several countermeasures is therefore a logical approach to counteract the multiple effects of cardiovascular deconditioning. The pressure gradient generated by LBNP also produces a form of artificial gravity and allows running exercise in the supine posture with normal footward loading (Hargens et al. 1991). Furthermore, treadmill exercise within LBNP provides lower body fluid redistribution and cardiovascular responses similar to upright exercise in $1 \mathrm{~g}$ environment (Murthy et al. 1994; Boda et al. 2000). A daily 40-min session of treadmill exercise within LBNP (LBNP/Ex) fails to protect orthostatic tolerance after a 15-day HDBR (Schneider et al. 2002). However, the addition of a 5-min period of LBNP without exercise immediately after the exercise period attenuates the loss of orthostatic tolerance after a 30-day HDBR (Watenpaugh et al. 2007). Thus, increasing the resting LBNP period after exercise from 5 to $10 \mathrm{~min}$ may further preserve orthostatic tolerance during HDBR by providing additional stimulation of orthostatic reflexes.

This paper reports the results from a part of an extensive WISE study (for Women International Space Simulation for Exploration) which was conducted to evaluate whether the combination of an aerobic treadmill exercise within LBNP and resistive exercise performed on a flywheel ergometer prevents physiological deconditioning in women during 60 days of HDBR. Association of a resistive flywheel exercise to the LBNP/Ex countermeasure was justified by the positive effects obtained on bone and muscle with resistive exercise after a 90-day bed rest study in men (Rittweger et al. 2005; Alkner and Tesch 2004). Effects on bone, muscle and other aspects of this study (e.g., effects on female endocrine responses, aerobic capacity...) will be reported elsewhere. In this paper, we tested the hypothesis that aerobic exercise in a LBNP chamber followed by 10 min of resting LBNP, three to four times a week, combined with flywheel resistance training of the legs, three times a week, maintains orthostatic tolerance in women during a 60-day HDBR.

\section{Materials and methods}

\section{Subjects and general protocol}

Sixteen healthy adult women volunteered for this study after receiving a complete description of the experimental methods, and after passing medical and psychological screening criteria. Medical tests for selection included: medical history, clinical and psychological examination, chest X-ray, electrocardiogram, abdominal echography, ultrasound imaging of lower limb veins, a 10 min head-up tilt test (intolerant subjects were excluded), peak oxygen uptake measurement $\left(\mathrm{VO}_{2} \mathrm{pk}\right)$, DEXA measurement for bone density and body composition, and laboratory tests (haematology, blood chemistry, urine analysis). The study was approved by the local Ethics Committee (Comité Consultatif de Protection des Personnes dans la Recherche Biomédicale). Each volunteer signed a consent form and was aware of her right to withdraw from the experiment without prejudice at any time. Subjects were housed in the Institute for Space Physiology and Medicine (MEDES), Toulouse, France.

The experiment protocol included 20 days of ambulatory control period, followed by 60 days of $6^{\circ}$ HDBR and 21 days of rehabilitation. Subjects were housed in pairs and both members of the pair were in the same experimental group and performed each experiment or countermeasure on the same day. During the HDBR period, subjects were monitored by video to ensure their compliance with the 
head-down tilt position. During the control and rehabilitation periods, the subjects remained in the MEDES facility.

All women had a regular menstrual cycle, and oral contraception was stopped at least 2 months before the beginning of HDBR. Tobacco, coffee, tea and alcohol were prohibited during the stay at the clinic. Each subject was given a caloric intake of $110 \%$ of their resting metabolic rate which was determined prior to HDBR. The maximum liquid intake of the control group was $60 \mathrm{ml} / \mathrm{kg}$ per day, and the maximum liquid intake for the exercise group was $60 \mathrm{ml} / \mathrm{kg}$ per day on the days without training and $75 \mathrm{ml} / \mathrm{kg}$ per day on days with exercise training. Caloric intake was increased for the exercise subjects to compensate for the energy costs of the exercise countermeasures.

\section{Groups}

Two groups of eight volunteers were selected: a control group (CON, $n=8)$ and an exercise group (EX, $n=8)$. Subjects were assigned to the different groups such that no between-group difference existed in mean peak oxygen uptake $\left(\mathrm{VO}_{2} \mathrm{pk}\right)$. There was no significant difference pre HDBR between CON and EX groups, respectively, in age (years, mean $\pm \mathrm{SE}$ ): $34 \pm$ versus $33 \pm 1, P=0.46$, height $(\mathrm{cm}, \quad$ mean $\pm \mathrm{SE}): \quad 163 \pm 2$ versus $165 \pm 3, \quad P=0.49$, weight $(\mathrm{kg}, \quad$ mean $\pm \mathrm{SE}): \quad 56.5 \pm 1.2$ versus $59.5 \pm 2$, $P=0.34$, and $\mathrm{VO}_{2}$ pk $(\mathrm{ml} / \mathrm{min}$ per $\mathrm{kg}$, mean $\pm \mathrm{SE}): 38.9 \pm$ 6.8 versus $37.9 \pm 4.0, P=1.0$.

Volunteers of the EX group performed alternately two types of exercises:

(1) Aerobic exercise

Subjects ran on a vertical treadmill while supine in the LBNP chamber (Fig. 1) for $40 \mathrm{~min}$ each session, with 10 min of resting LBNP immediately after the run. A session was scheduled for each subject approximately every 3-4 days/week beginning on HDBR day 1, for a total of 29 sessions during the 60-day HDBR. Unfortunately, due to constraints of other tests near the end of the HDBR period, the last LBNP countermeasure session was scheduled 62-63 $\mathrm{h}$ before the end on the 60-day HDBR.

During supine treadmill exercise, LBNP $(48-55 \mathrm{mmHg})$ was applied to produce footward forces equivalent to those for upright running on Earth, initially at 1.0 time body weight, increasing progressively to 1.15 times body weight according to subject tolerance. The $\mathrm{VO}_{2}$ pk for each subject was determined with an upright, graded treadmill stress test before HDBR. The treadmill speeds used during exercise within the LBNP chamber were calculated from a linear regression of the oxygen consumption and exercise intensity results from the pre $\mathrm{HDBR} \mathrm{VO}_{2}$ pk test. The interval exercise protocol was similar to previous experiments (Watenpaugh et al. 2000; Schneider et al. 2002; Watenpaugh

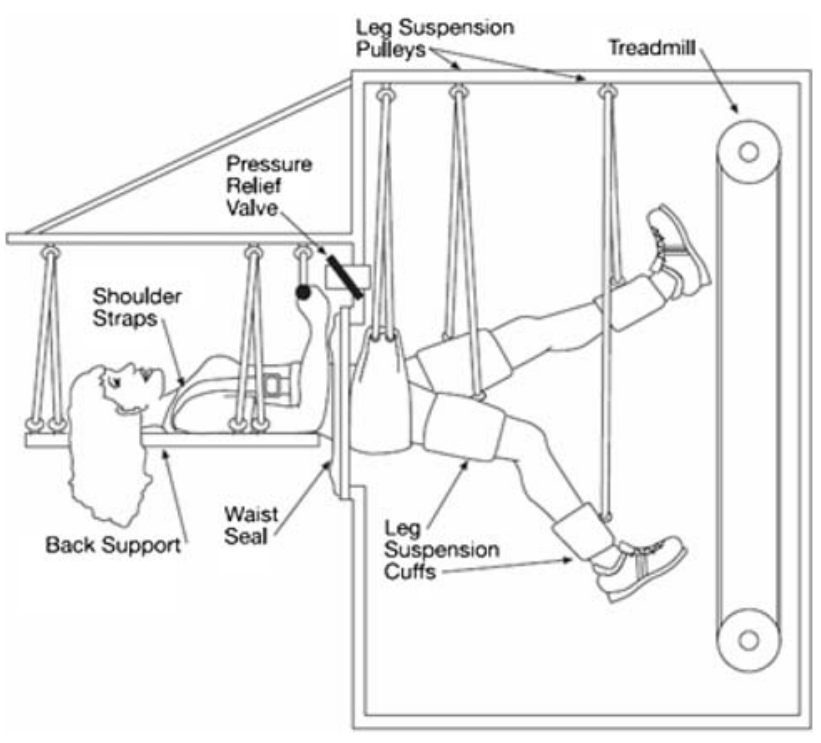

Fig. 1 Exercise on a treadmill within lower body negative pressure (LBNP) exposure. Footward force is produced by the pressure gradient generated by LBNP. The subject is suspended at the ankles and thighs by a pulley system, and the hands are holding the suspension cables at waist level

et al. 2007): 7 min to warm up at $40 \% \mathrm{VO}_{2} \mathrm{pk}$, followed by $3 \mathrm{~min}$ at $60 \%, 2 \mathrm{~min}$ at $40 \%, 3 \mathrm{~min}$ at $70 \%, 2 \mathrm{~min}$ at $50 \%$, $3 \mathrm{~min}$ at $80 \%, 2 \mathrm{~min}$ at $60 \%, 3 \mathrm{~min}$ at $80 \%, 2 \mathrm{~min}$ at $50 \%$, $3 \mathrm{~min}$ at $70 \%, 2 \mathrm{~min}$ at $40 \%, 3 \mathrm{~min}$ at $60 \%$ and $5 \mathrm{~min}$ at $40 \% \mathrm{VO}_{2} \mathrm{pk}$, with an additional $10 \mathrm{~min}$ of supine, stationary exposure to the same level of LBNP (resting LBNP period).

Fatigue was evaluated by the subject at the end of each stage using a visual analog scale, from 6 (very easy) to 20 (exhaustion). Any score above 18 led to immediate reduction of the treadmill speed, in order to stay at a sub-maximal effort. Heart Rate (HR) was continuously monitored by a Polar $^{\circledR}$ (Polar Electro Inc., NY, USA) heart rate watch with the transmitter belt fixed around the chest.

Subjects were monitored closely during the resting LBNP period: systolic and diastolic blood pressures (SBP and DBP) were measured continuously by a non-invasive finger cuff (Finapres 2300, Ohmeda France) on the right hand placed at heart level, with a correction of the finger blood pressure every minute by comparing to an automatic arm blood pressure device (Dynamap Monitor, Critikon Inc., Tampa, Fla, USA) on the left arm. Pain, discomfort or signs of pre-syncope were noted, and rated on a visual scale from 1 (slight symptom) to 10 (maximal intensity for symptom). If symptoms appeared, the volunteer was asked to move her legs to improve venous return, and a cold wet towel was applied to her face. The LBNP was turned off if one of the following signs occurred: rapidly increasing symptoms (nausea, clammy skin, excessive sweating, pallor, vertigo), a sudden drop in blood pressure (SBP 
fall $>25 \mathrm{mmHg} / \mathrm{min}$ or DBP fall $>15 \mathrm{mmHg} / \mathrm{min}$ ), a $\mathrm{SBP}<70 \mathrm{mmHg}$, a sudden drop in HR (HR fall $>15$ beats/ $\mathrm{min})$. Once symptoms had disappeared, the resting LBNP period was resumed.

(2) Resistive exercise

Subjects trained the back, thigh and calf muscle groups using supine squat (SS) and calf press (CP) exercises using a gravity independent inertial ergometer (Alkner and Tesch 2004). A total of 19 sessions were scheduled for each subject approximately every third day (2-3 days/week) beginning on day 2 of HDBR. The inertial ergometer was in the $6^{\circ}$ head-down tilt position and all resistance exercise was performed in this position. The SS exercise consisted of 4 sets of 7 maximal concentric and eccentric repetitions, while the $\mathrm{CP}$ exercise consisted of 4 sets of 14 maximal concentric and eccentric repetitions. This resistive exercise protocol was identical to a previous 90-day HDBR study conducted in males (Alkner and Tesch 2004).

\section{Orthostatic tolerance test}

Orthostatic tolerance was assessed by a tilt/LBNP test. This tilt/LBNP test was performed twice before HDBR (the first one to familiarize subjects with their presyncopal symptoms, the second one as a baseline pre HDBR test), and once immediately at the end of HDBR period (recovery day 1). Subjects were positioned and secured on a tilt table, the lower part of the body inside a LBNP chamber. LBNP was applied by enclosing the subject's lower body up to the iliac crest in an air tight box. A footplate allowed for foot contact to maintain body position. The subject was asked to avoid leg movements during the test.

After $5 \mathrm{~min}$ in the supine position for collection of baseline data, the test consisted of $5 \mathrm{~min}$ supine rest, $10 \mathrm{~min} 80^{\circ}$ head-up tilt without LBNP, followed by continued head-up tilt and progressively increasing stages of LBNP of $3 \mathrm{~min}$ each $(10,20,30 \mathrm{mmHg}$, etc.) until presyncope. This test allows a precise and reproducible assessment of orthostatic tolerance (el-Bedawi and Hainsworth 1994). The test was ended if one of the following signs occurred: rapidly increasing symptoms (nausea, clammy skin, excessive sweating, pallor, vertigo), a sudden drop in blood pressure ( SBP fall $>25 \mathrm{mmHg} / \mathrm{min}$ or DBP fall $>15 \mathrm{mmHg} / \mathrm{min}$ ), a $\mathrm{SBP}<70 \mathrm{mmHg}$, a sudden drop in HR (HR fall $>15$ beats/ min), cardiac dysrhythmias.

During these tests, HR was obtained by standard electrocardiography and SBP and DBP were measured continuously with a non-invasive finger cuff method (Finometer ${ }^{\circledR}$, Finapress Medical Systems, The Netherlands). A heightcorrector placed at the level of the heart provided a stable measurement independent of hand movement, position or effect of tilting. Resting blood pressure values obtained with the finger cuff were calibrated to SBP and DBP values obtained with an inflatable arm cuff. Mean arterial pressure (MAP) was determined as the average value over a complete cardiac cycle. Cardiac output (Q) was estimated from the Modelflow method (Wesseling et al. 1993), which computes an aortic flow waveform from finger pressure, by simulating a non-linear three-element model of the aortic input impedance. The computed aortic flow waveform per beat provided left ventricular stroke volume (SV) and consequently $\mathrm{Q}$, by multiplying SV by instantaneous HR. An estimate of total peripheral resistance (TPR) was calculated from MAP/Q. Considering the limitations of the Modelflow method in providing absolute values of $\mathrm{SV}, \mathrm{Q}$ and consequently SVR (Pitt et al. 2004), these variables were presented as percent changes from supine resting values (for more details, see limitations in "Discussion").

\section{Blood volume}

Blood volume (BV) was calculated in all subjects 1 day before tilt/LBNP tests pre HDBR and on day 60 of HDBR, by carbon monoxide (CO) rebreathing as described by Burge and Skinner (1995), which was shown to provide a safe, precise and reproducible estimation of blood volume in healthy volunteers.

\section{Statistical analysis}

Orthostatic tolerance time (OT time), hemodynamic variables during OT tests, and BV were compared in each group and between groups before and after HDBR. Hemodynamic variables obtained during tilt/LBNP tests pre and post HDBR (HR, SV, Q, SBP, DBP, MAP, TPR) were averaged in each group over the last $30 \mathrm{~s}$ of the fourth minute of test for the supine values and over the last $30 \mathrm{~s}$ of each minute during the tilt and tilt/LBNP periods. Due to onset of presyncopal symptoms, the number of subjects completing each tilt/LBNP stage decreased over time in each group: hemodynamic variables were compared every minute as long as there were data from six subjects per group. Normality of variables was tested by KolmogorovSmirnov test. Student's paired $t$ test was used to compare OT time and BV pre to post HDBR in EX and CON groups, and unpaired $t$ test was used to compare OT time and $\mathrm{BV}$ between groups before and after HDBR. Two way analysis of variance (ANOVA) was used to determine the effects of group, HDBR, and group X HDBR interaction for hemodynamic variables during tilt/LBNP tests. An SPSS/ $\mathrm{PC}+$ software package was used for statistical analyses (version 15.0, SPSS, Chicago, IL). Values are expressed as mean \pm SE. Statistical results were considered significant when $P<0.05$. 


\section{Results}

\section{Compliance of exercisers to resting LBNP period}

Seven out of eight exercisers completed at least 19 of the 29 resting LBNP sessions at the end of exercise without any break or reduction in LBNP. Conversely, the relatively low compliance of one exerciser to resting LBNP periods forced reduction of the level of LBNP, providing a very short time of training effectively achieved at a LBNP level simulating one body weight $(6 \%)$. Another subject had also a low compliance to the resting LBNP period, and completed $55 \%$ of the resting LBNP time at a LBNP level simulating one body weight. The other six exercisers completed $88-100 \%$ of the prescribed resting LBNP regimen.

According to the general agreement between WISE principal-investigators in December 2005 at an Investigator Working Group meeting in Montreal, results are given and analyzed with all the subjects included.

\section{Orthostatic tolerance time}

Our combined tilt/LBNP procedure provided a clear endpoint for all tests, pre and post HDBR. In all cases, tilt/ LBNP tests were ended because of a sudden, rapid fall in SBP, associated with a drop in HR in five of seven EX and four of eight CON. OT time (mean $\pm \mathrm{SE}$ ) decreased from $17.5 \pm 1.0 \mathrm{~min}$ to $9.1 \pm 1.5 \mathrm{~min}$ in CON group $(-50 \pm 6 \%$, $P<0.001)$, and from $19.3 \pm 1.3 \mathrm{~min}$ to $13.0 \pm 1.9 \mathrm{~min}$ $(-35 \pm 7 \%, P<0.001)$ in EX group. There was no significant difference in OT time between groups before HDBR $(P=0.30)$ and at the end of $\operatorname{HDBR}(P=0.13)$ (Fig. 2, left).
Hemodynamic variables during tilt/LBNP tests

Mean values of hemodynamic variables at presyncope were calculated and plotted on graphs to emphasize mean changes of OT time and hemodynamic variables at the presyncopal end point in CON and EX group before and after 60 days HDBR. Curves were drawn as long as there were at least six subjects per group: $n=8$ at the beginning of tilt/ LBNP tests in both groups, decreasing progressively related to occurrence of presyncope.

Heart rate (Fig. 3): Pre HDBR, HR (bpm, mean \pm SE) was similar in $\mathrm{CON}$ and $\mathrm{EX}$ groups at rest $(71 \pm 3$ and $68 \pm 3$, respectively) and over the course of the tilt/LBNP tests, with a similar increase in the two groups after the onset of upright posture. At presyncope, HR had reached similar levels in the two groups $(127 \pm 7$ in CON, $135 \pm 6$ in EX).

The post HDBR supine HR in CON was significantly elevated compared to pre HDBR value ( $85 \pm 4$ vs. $71 \pm 3$, $P<0.01)$, whereas it was not the case in EX $(75 \pm 5$ vs. $68 \pm 3$, NS). Over the course of the tilt/LBNP tests, a significant increase in HR compared to pre HDBR values was observed in the two groups (main effect of HDBR, $P<0.01)$. This HR increase was more pronounced in CON compared to EX group, and the difference became significant after $3 \mathrm{~min}$ in upright posture with a HR of $129 \pm 4$ in CON and $115 \pm 4$ in EX group (significant group X HDBR interaction effect, $P<0.05)$. At presyncope, HR had reached similar levels in the two groups $(135 \pm 6$ in CON, $133 \pm 8$ in EX).

Blood pressure (Fig. 4): SBP and DBP (mmHg, mean $\pm \mathrm{SE}$ ) in CON and EX groups were not significantly different before HDBR at supine rest and over the course of
Fig. 2 Left orthostatic tolerance time (min, mean $\pm \mathrm{SE}$ ) assessed by tilt/LBNP tests pre and post HDBR in CON and EX groups. There was no significant difference between groups pre HDBR, $17.5 \pm 1 \mathrm{~min}$ in CON and $19.3 \pm 1.3 \mathrm{~min}$ in EX, respectively $(P=0.30)$. Orthostatic tolerance time significantly decreased post HDBR in EX and CON groups, $13.0 \pm 1.9 \mathrm{~min}$ and $9.1 \pm 1.5 \mathrm{~min}$, respectively $(* P<0.001)$. Right blood volume assessed by $\mathrm{CO}$ rebreathing method pre and post HDBR in $\mathrm{CON}$ and EX groups. BV decreased significantly in CON $(-9 \pm 2 \%, P<0.01)$, with a non-significant decrease in EX $(-4 \pm 3 \%, P=0.17)$
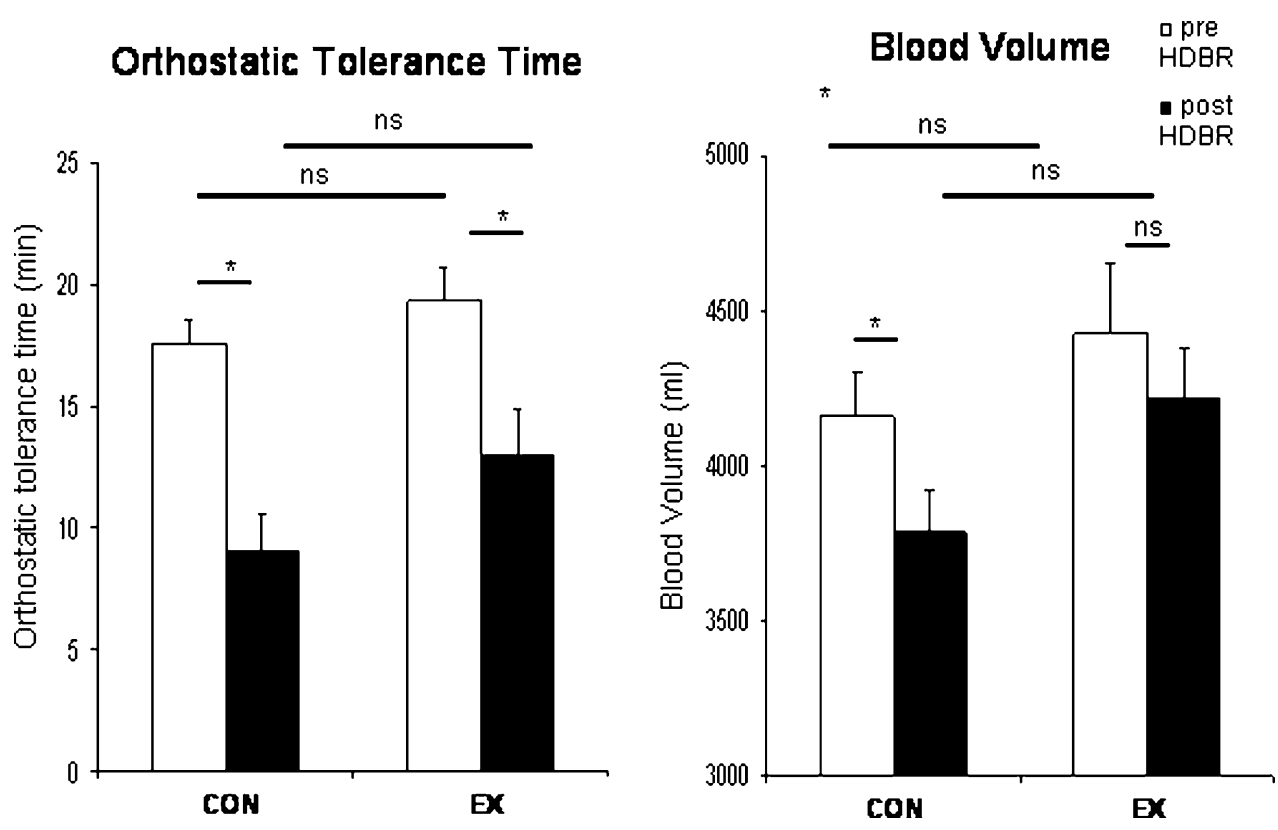


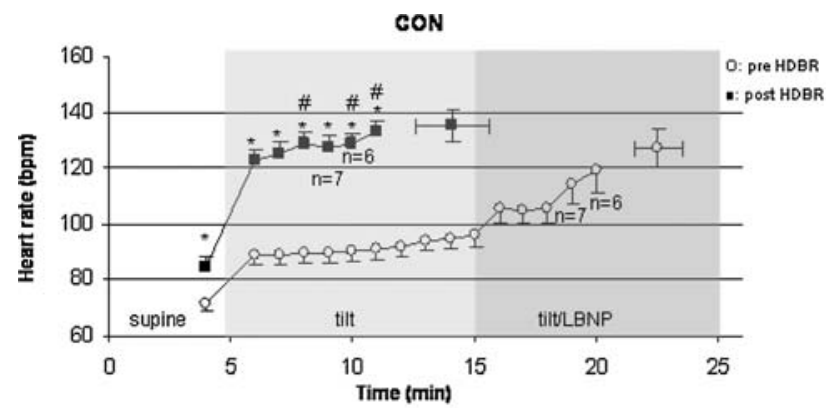

EX

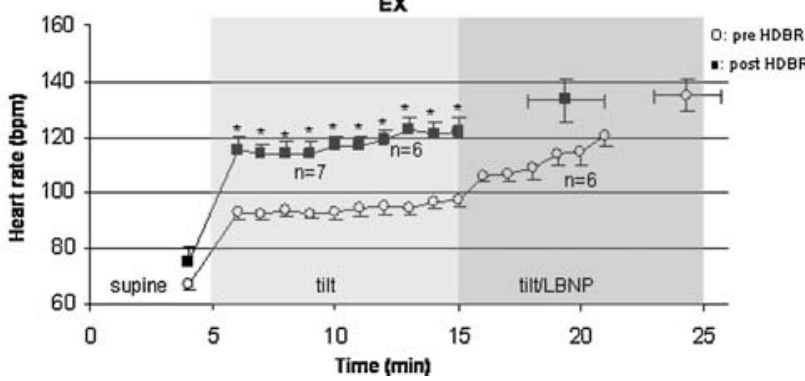

Fig. 3 Heart rate during tilt/LBNP tests (beats per min, mean \pm SE) are shown for the CON and EX conditions, before (open circles) and after (closed squares) HDBR. Isolated points are HR at presyncopal time points. *Indicates significantly higher values post HDBR compared with pre HDBR values. "Indicates a significantly higher HR in CON compared with EX at the same time point of the post HDBR tilt/ LBNP test
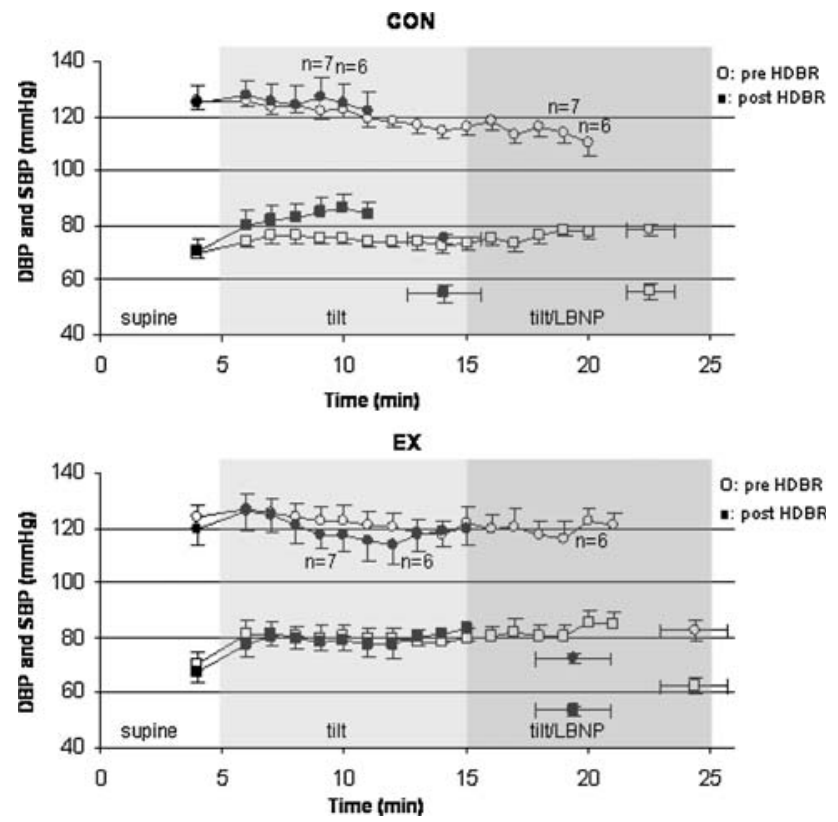

Fig. 4 SBP (top) and DBP (bottom) during tilt/LBNP tests (mmHg, mean $\pm \mathrm{SE}$ ) are shown for the CON and EX conditions, before (SBP: open circles, DBP: open squares) and after (SBP: close circles, DBP: closed squares) HDBR period. Isolated points are SBP and DBP at presyncopal time point pre and post HDBR

the tilt/LBNP tests. Pre HDBR presyncopal values of SBP were $78 \pm 2$ and $82 \pm 4$ for CON and EX group, respectively, $56 \pm 3$ and $63 \pm 3$ for DBP. Supine and tilt blood
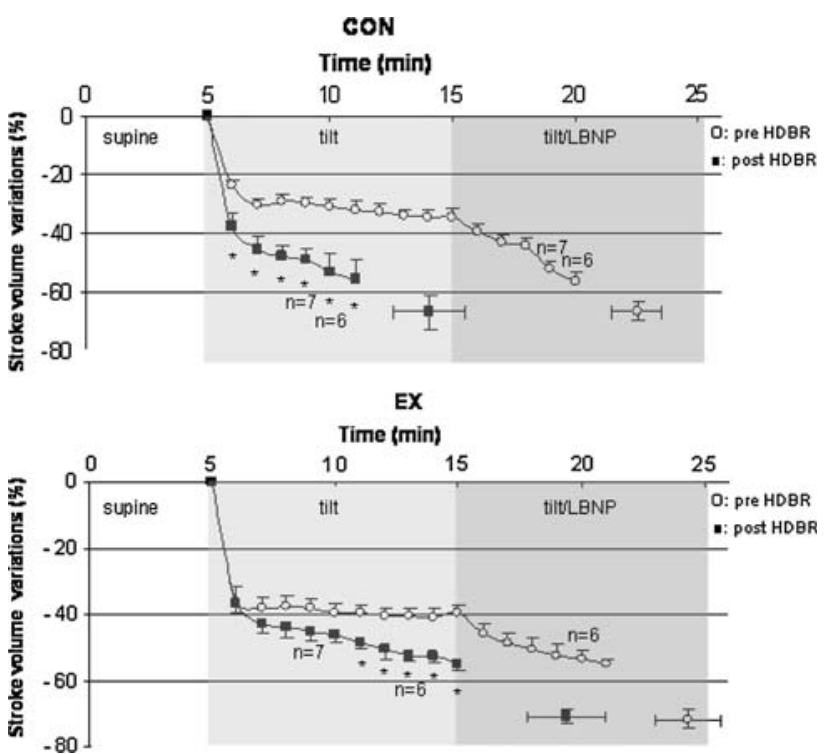

Fig. 5 Stroke volume changes during tilt/LBNP tests (\%, mean \pm SE) are shown for the CON and EX conditions, before (open circles) and after (closed squares) HDBR. Isolated points are changes in SV at presyncopal time point pre and post HDBR. *Indicates significant decrease in SV values post HDBR compared with pre HDBR values

pressure values were not significantly affected by HDBR, and were not different between groups, except for the earlier occurrence of presyncopal symptoms in CON group. Presyncopal blood pressure values were not different: $75 \pm 2$ and $72 \pm 2$ in CON and EX groups for SBP, $55 \pm 3$ and $54 \pm 2$ for DBP, respectively.

Stroke volume (Fig. 5): pre HDBR, the decrease in SV $(\%$, mean $\pm \mathrm{SE})$ was similar in CON and EX over the course of the tilt/LBNP tests $(-34 \pm 3 \%$ vs. $-40 \pm 3 \%$ at $10 \mathrm{~min}$ in upright posture, NS) and at presyncope $(-66 \pm 4 \%$ in CON, $-72 \pm 3 \%$ in EX, NS). In CON group, the main effect of HDBR was a fall in SV since the onset of upright posture $(-38 \pm 4 \%$ vs. $-24 \pm 2 \%$ at $1 \mathrm{~min}, P<0.01,-53 \pm 7$ vs. $-31 \pm 2$ at $5 \mathrm{~min}, P<0.05$ ). In EX group post HDBR, the decrease in SV over the course of the tilt/LBNP test was significantly more pronounced than pre HDBR since $6 \mathrm{~min}$ in upright posture $(-49 \pm 2 \%$ vs. $-40 \pm 2 \%, P<0.05)$. A statistically significant group $\mathrm{X}$ HDBR interaction effect was observed since $4 \mathrm{~min}$ in upright posture, $P<0.05$ ). At presyncope, the fall in SV was similar in the two groups $(-67 \pm 5 \%$ in CON group, $-71 \pm 2 \%$ in EX group, NS).

Cardiac output (Fig. 6): pre HDBR, the decrease in cardiac output $\mathrm{Q}(\%$, mean $\pm \mathrm{SE})$ was similar in $\mathrm{CON}$ and $\mathrm{EX}$ groups over the course of the tilt/LBNP tests $(-11 \pm 4 \%$ vs. $12 \pm 5 \%$ at $10 \mathrm{~min})$ and at presyncope $(-42 \pm 4 \%$ vs. $-44 \pm 5 \%, \mathrm{NS}$ ). HDBR did not affect Q in CON and EX groups, and no HDBR X group interaction was observed.

Total peripheral resistance (Fig. 7): pre HDBR, TPR (\%, mean \pm SE) increased similarly in CON and EX groups 


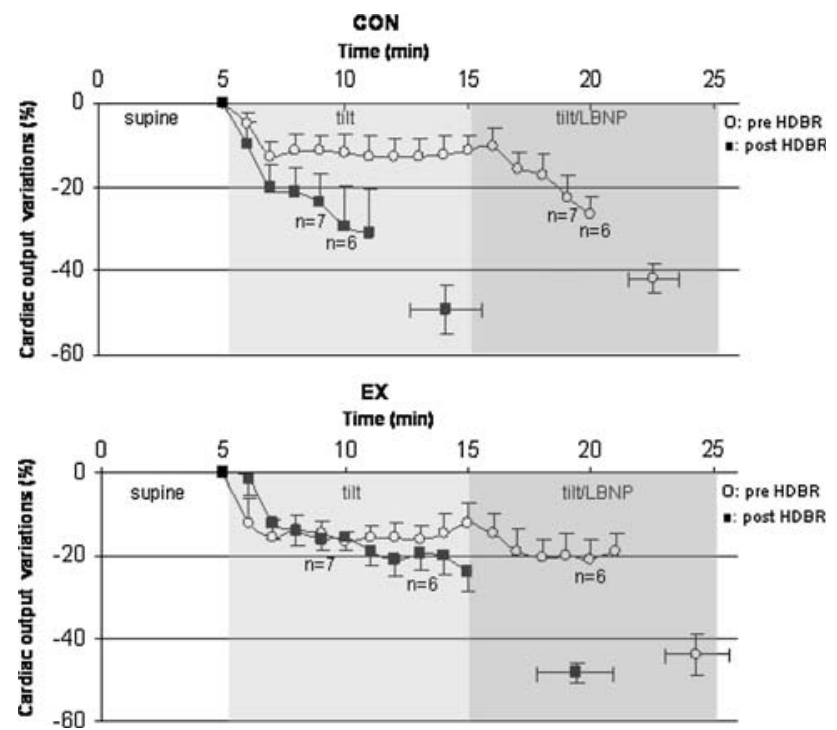

Fig. 6 Cardiac output changes during tilt/LBNP tests are shown for the CON and EX conditions, before (open circles) and after (closed squares) HDBR. Isolated points are changes in $\mathrm{Q}$ at presyncopal time point pre and post HDBR. No significant change in $Q$ was observed pre to post HDBR in CON and EX groups

over the course of the tilt/LBNP tests $(+14 \pm 6 \%$ vs. $+23 \pm 6 \%$ at $10 \mathrm{~min}, \mathrm{NS})$ and at presyncope $(+26 \pm 10 \%$ vs. $+44 \pm 11 \%$, NS). Significant HDBR and HDBR X group interaction effects $(P<0.05)$ were observed after the fourth minute in head-up tilt posture. In the CON group post HDBR, the increase in TPR was more pronounced than pre HDBR $(+89 \pm 32 \%$ vs. $+18 \pm 7 \%$ at $5 \mathrm{~min}$, $P<0.05)$. In the EX group, the post HDBR increase in TPR over the course of the tilt/LBNP tests was not significantly different than pre HDBR $(+27 \pm 3 \%$ vs. $+29 \pm 5 \%$ at 5 min in upright posture, NS, $+42 \pm 11 \%$ vs. $+23 \pm 6 \%$ at $10 \mathrm{~min}, \mathrm{NS})$. At presyncope, the increase in TPR was not different in the two groups $(+54 \pm 20 \%$ in CON group, $+39 \pm 9 \%$ in EX group, NS).

Blood volume

A technical problem (clotted blood sample) with the BV determination in one subject from the EX group at the end of HDBR led us to exclude this subject from analysis. There was no significant difference in BV between groups pre HDBR. In CON, BV (mean \pm SE) decreased from $4,161 \pm 142 \mathrm{ml}$ pre HDBR to $3,783 \pm 136 \mathrm{ml}$ on day 60 of HDBR $(-9 \pm 2 \%, P<0.01)$. In EX group, BV was maintained $(4,427 \pm 224 \mathrm{ml}$ before HDBR, 4,213 $\pm 168 \mathrm{ml}$ on day 60 of HDBR, $-4 \pm 3 \%, P=0.17$ ) (Fig. 2, right).

\section{Discussion}

The combined exercise countermeasure used in this study fails to protect orthostatic tolerance in women after a 60-day HDBR: OT time is not different after HDBR in CON and
Fig. 7 Total peripheral resistance changes $(\%$, mean $\pm \mathrm{SE})$ during tilt/LBNP tests are shown for the CON and EX conditions, before (open circles) and after (closed squares) HDBR. Isolated points are TPR at presyncopal time point pre and post HDBR. *Indicates significant increase in TPR values post HDBR compared with pre HDBR values
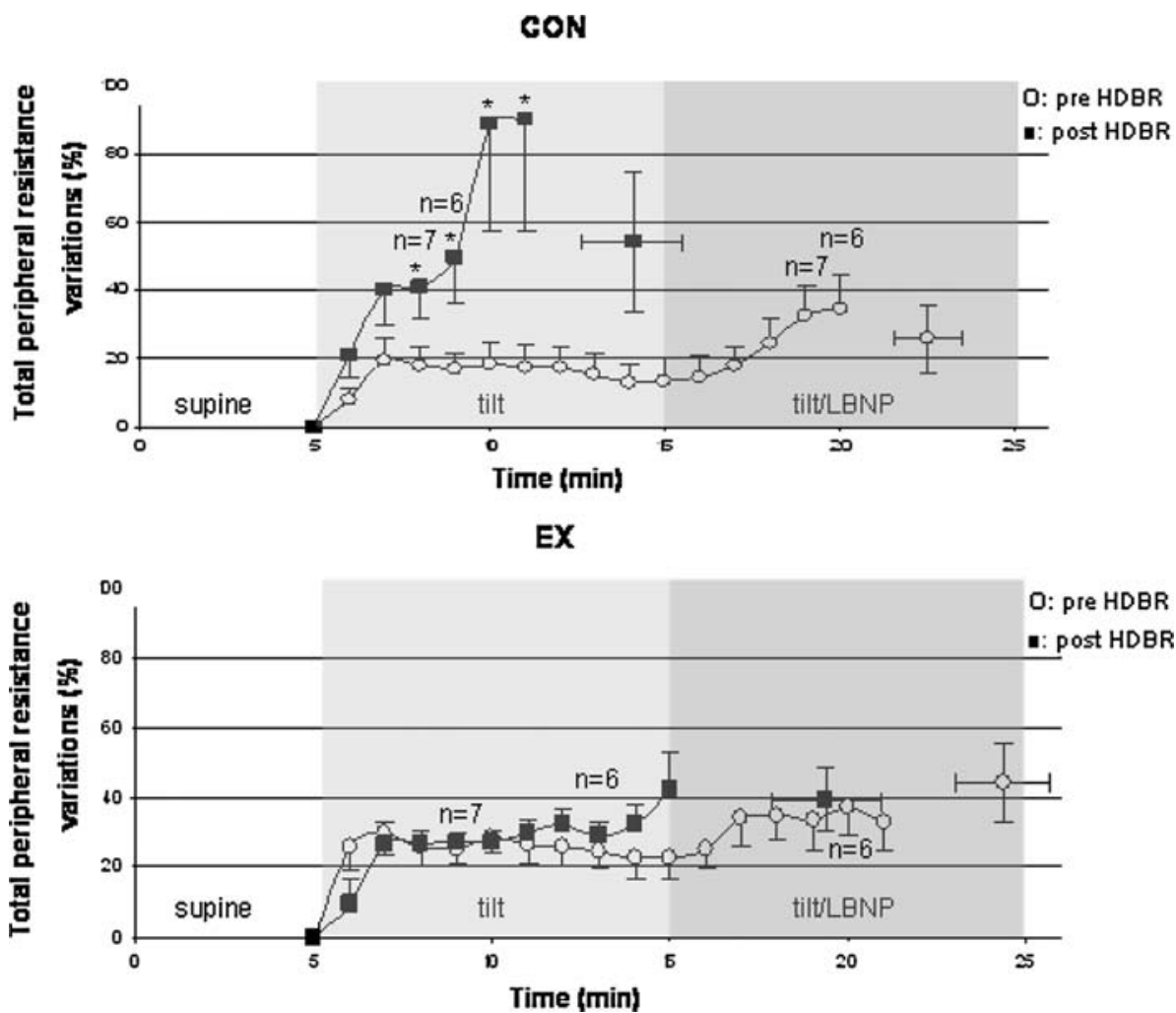
EX groups. Nevertheless, some cardiovascular improvements in the EX group post HDBR are noteworthy: supine $\mathrm{HR}$ is maintained during HDBR, HR is lower and SV is better maintained over the course of the tilt/LBNP test. Furthermore, BV is maintained in EX and decreases significantly in CON.

Very few data exist on cardiovascular response to headup tilt in women after long duration spaceflight or HDBR. After a 120-day HDBR in women, Maillet et al. (2000) observe a better maintained $\mathrm{BP}$ and a slower increase in $\mathrm{HR}$ during a 20 min head-up tilt test in a countermeasure group (60-80 min of daily supine running exercise on a treadmill with bungee cords compared to a control group $(n=4$ in each group). The greater HR response to an orthostatic challenge observed in their control group is also noted in our study, supporting an intact or even enhanced cardiac arm of the baroreflex. Concerning the vascular arm of the baroreflex, it is noteworthy that (1) the increase in TPR is more pronounced post HDBR in CON group than pre HDBR, (2) this maximal vascular response occurs earlier than pre HDBR, and (3) this response can not be maintained as long as pre HDBR, especially in the CON group. An early drop in SV is transiently compensated by a pronounced increase in HR (changes in Q are not significant), MAP is initially maintained by increased TPR (MAP = $(\mathrm{SV} \times \mathrm{HR}) \times \mathrm{TPR})$, but these responses are rapidly overwhelmed resulting in early presyncope (Figs. 4, 5, 6, 7). The mechanisms involved in this altered response to orthostasis remain unclear but may include decreased blood volume, cardiac and vasculature remodelling, central nervous system dysregulation, and decreased ability to mobilise blood volume from the splanchnic area.

We did observe a maintained BV in our EX group and a significant loss after HDBR in our CON group, although less pronounced than in some previous HDBR studies of male subjects. A decrease in plasma volume of $10-20 \%$ over the first 30 days is a consistent finding for HDBR studies ranging from 30 to 90 days in male volunteers (Fortney et al. 1988, 1994; Traon et al. 1995; Custaud et al. 2002; Belin de Chantemele et al. 2004a). There have been few previous investigations of the effects of HDBR on $\mathrm{BV}$ in women compared to men, and all such studies were of relatively short duration. A similar reduction of $\sim 9 \%$ in male and female subjects was reported after a 7-day HDBR study (Custaud et al. 2002), while Fortney et al. reported less reduction of blood volume in women than men $(-10 \%$ vs. $-15 \%)$ after a 13-day HDBR study (Fortney et al. 1994). A complicating factor in the present study in the comparison between groups was that $\mathrm{BV}$ was measured on HDBR day 60 which was approximately $36 \mathrm{~h}$ after the last LBNP/Ex countermeasure session. BV is known to be reduced within the first $24 \mathrm{~h}$ of HDBR (Vernikos et al. 1993). Therefore, the differences between groups might have been larger if measured on a similar schedule to that in other studies.

Concerning cardiac remodelling, it was found by Dorfman et al. (2007) in the current WISE study that left and right ventricular volumes were maintained and left and right ventricular masses were increased in EX, compared to significant decreases in CON. Cardiac remodelling and atrophy (Levine et al. 1997; Dorfman et al. 2007) likely contributes to the reduced SV observed in $\mathrm{CON}$ in our study.

Several other factors may play a role in the better maintained cardiovascular responses in EX. Arbeille et al. in this WISE study, document that the combined exercise countermeasure attenuates the bed rest effect on leg vein capacitance, with an increased pooling of blood in lower limbs veins in CON subjects compared with EX during an orthostatic stress (Arbeille et al. 2008). Edgell et al. observe a decreased basal leg vascular resistances in non-exercising subjects, whereas EX subjects have increased LVR post HDBR (Edgell et al. 2007). Last, Demiot et al. demonstrate an endothelial dysfunction in CON, with a reduced endothelium dependant vasodilation and an increased number of circulating endothelial cells, whereas those changes are not observed in EX (Demiot et al. 2007). All these factors are implicated in a better maintained venous return to the heart during an orthostatic stress in EX through a maintained vascular reactivity, resulting in the slower decrease in stroke volume.

Interestingly, the results of EX group in our study show that despite maintained cardiac mass, maintained BV and attenuated cardiovascular changes associated with an orthostatic stress, orthostatic intolerance was not prevented. In our EX group, decreased OT while BV is maintained underlines the fact that $\mathrm{BV}$ is not a main factor for OT, at least after a long duration HDBR. Furthermore, in a retrospective analysis of contributory factors to orthostatic intolerance in men, Pavy-Le-Traon et al. observe that a reduced plasma volume is found in many tolerant subjects to orthostatism after HDBR (Pavy-Le Traon et al. 1999).

Our OT time result is in opposition with a previous study, where a 40 min of treadmill LBNP/Ex followed by 5 min of resting LBNP, 6 days/week, resulted in significantly less reduction in OT time in EX compared with CON $(-13 \%$ vs. $-34 \%)$ (Watenpaugh et al. 2007). Nevertheless, several differences exist between the earlier 30 day HDBR study and WISE. These factors are discussed below: (1) the HDBR period was longer (60 days), (2) WISE was conducted on female volunteers only, (3) WISE combined a resistive exercise and an aerobic exercise within LBNP, resulting in a decreased number of LBNP/Ex sessions (three to four per week for WISE compared with six per week for the 30 day HDBR study) (4) resting LBNP period after exercise was increased from 5 to $10 \mathrm{~min}$ in WISE 
(5) the OT test was scheduled $24 \mathrm{~h}$ after the last LBNP/Ex session in the study of Watenpaugh et al. (2007), but our OT test took place $62-63 \mathrm{~h}$ after the last LBNP/Ex session in WISE (6) the sample size was smaller in WISE with 8 subjects per group compared with 15 per group in the 30-day HDBR study.

Increased duration of bed rest or spaceflight is associated with higher incidence of orthostatic intolerance (Meck et al. 2001), and women are more susceptible to orthostatic intolerance than men (Convertino 1998; Fu et al. 2004).

The resistive exercise performed by EX group during WISE was similar to the one performed every third day during a previous 90-day HDBR study in male subjects. This exercise showed no beneficial effect on orthostatic tolerance (Belin de Chantemele et al. 2004a) and did not prevent HDBR-induced reduction in blood volume (Belin de Chantemele et al. 2006). It is therefore unlikely that flywheel resistive exercise plays an important role in orthostatic tolerance in our female subjects.

It had been suggested by Watenpaugh et al. that increasing the resting LBNP period at the end of LBNP/Ex sessions might preserve OT (Watenpaugh et al. 2007). However, the poor compliance of one subject of EX group to the resting LBNP period after exercise led to decrease the level of LBNP applied, thus compromising the countermeasure efficacy. It is noteworthy that exclusion of this non-compliant subject from analysis results in significantly less reduction in OT time after HDBR in EX compared with $\mathrm{CON}(-28 \pm 5 \%$ vs. $-50 \pm 7 \%, P=0.02)$. Therefore, the benefit for orthostatic intolerance of the resting LBNP period after exercise must be balanced with the risk of presyncope. Careful monitoring, especially during the first few training sessions, is necessary to familiarize each subject with: (1) their own presyncopal symptoms, (2) the effect of leg muscle contraction on such signs and (3) manipulation of an emergency valve to stop LBNP in case of increasing symptoms. On the other hand, the beneficial effect on orthostatic tolerance of this resting LBNP period may be related to the regular onset of a nearly presyncopal state (Lightfoot et al. 1989). The intensity, duration and frequency of orthostatic stress should probably be adapted to each subject.

Considering the lack of impact of the flywheel countermeasures in male subjects on orthostatic tolerance (Belin de Chantemele et al. 2004a) and the slight effect of LBNP/ exercise alone (Schneider et al. 2002), it is likely that the resting LBNP period after exercise plays a pivotal role for improved cardiovascular responses against an orthostatic stress in EX compared with CON in our study. Mechanisms involved are extensively described in a previous paper from Watenpaugh et al. (2007). Briefly, application of an orthostatic stimulus after exercise might stimulate an increased response of the sympathetic system to maintain blood pressure: post exercise changes could thus potentiate the cardiovascular stress imposed by the resting LBNP period. Recent work by Kimmerly et al. (2007) report a greater increase in muscle sympathetic nerve activity in subjects exposed to LBNP $(-35 \mathrm{mmHg}$ ) after a single bout of dynamic exercise compared with subjects without exercise.

Improvements in cardiovascular responses to tilt/LBNP tests in EX compared with CON group occurred even though the final exercise countermeasure session was performed $62-63 \mathrm{~h}$ before the final orthostatic tolerance test. Butler et al. have found that only $4 \mathrm{~h}$ of HDBR modifies cardiovascular responses to head-up tilt, with a marked increase in occurrence of presyncopal symptoms before 10 min of head-up tilt (Butler et al. 1991). Khan et al. observe that after $24 \mathrm{~h}$ of HDBR, the ability to augment the muscular sympathetic nerve activity at high levels of LBNP is reduced (Khan et al. 2002). Also, a recent study by Fischer et al. document an altered hormonal or neural control of regional vascular resistances after only $4 \mathrm{~h}$ of HDBR: a reduced ability to increase splanchnic vascular resistance was observed during an orthostatic stress, that could explain the decrease in venous return and consequently in stroke volume (Fischer et al. 2007). These studies reveal the fast adaptation of the cardiovascular system to new gravitational conditions. For this present long-term HDBR study in women, numerous tests were scheduled during the last days of the HDBR period, preventing any exercise during the 2.5 days before our post HDBR tilt/LBNP orthostatic tolerance test. A better maintained orthostatic tolerance in EX may have been possible by scheduling the countermeasure closer to the end of HDBR.

From the study by Watenpaugh et al. (2007) and this WISE study we can formulate some conclusions: (1) 10 min of resting LBNP after exercise at a LBNP level simulating one BW cannot be recommended because of a poor compliance in some subjects, (2) LBNP/Ex sessions with short post exercise resting LBNP periods should be scheduled during the very last days of HDBR or spaceflight, (3) repeated short periods of orthostatic stress during exercise may improve the efficacy of the countermeasure, especially during the last sessions (e.g., $5 \mathrm{~min}$ every $15 \mathrm{~min}$ of exercise) and (4) a short period of orthostatic stress after other modes of exercise (e.g., exercise associated with centrifugation) also may be beneficial.

Limitations of the study

An important factor that requires consideration in this study is the timing of experimentation with respect to studies of women and their menstrual phase. No subject was taking oral contraceptives for at least 2-months prior to the start of the research. In such a large study where subjects were housed in pairs and scheduling of multiple experiments was 
required, it is extremely difficult to balance or control the timing of an individual experiment with respect to menstrual cycle phase. As an indication of the phase of the cycle relative to experimentation, the number of subjects in the first 10 days of their cycle during the tilt/LBNP experiments was four CON and five EX during pre-HDBR and four CON and four EX at the end of HDBR. For blood volume experiments the corresponding numbers were three CON and three EX during pre HDBR testing and two CON and three EX on HDBR 60. Menstrual cycle phase does have an impact on plasma volume (Fortney et al. 1988) that might have masked the effect of HDBR and countermeasures in the current study. Differences between CON and EX groups probably did not skew the results as the number of subjects early in their cycle was similar in both groups. Nevertheless, menstrual cycles were altered in many subjects, and the certain phase of their cycle could only be definitively affirmed with plasma sex hormone dosages. Rather, the period of time between the last countermeasure and the measurements of blood volume or orthostatic tolerance due to the requirement for other experimentation within this large-scale project probably reduced betweengroup differences.

Another limitation concerns the accuracy of the Modelflow method to assess absolute SV from finger blood pressure, and consequently Q and TPR. Controversial data exist on the reliability of absolute SV data estimated by Modelflow from non-invasive finger pressure during orthostasis. A recent study, comparing SV obtained from Modelflow (using Portapres) versus a rebreathing method, indicates that Modelfow overestimates Q and has large inter-individual variations (Pitt et al. 2004). However, Harms et al. observe a non-significant offset of SV by Modelflow for a $70^{\circ}$ head-up tilt compared to the direct thermodilution method (Harms et al. 1999). Without an invasive calibrating method, absolute individual $\mathrm{SV}$ values should be viewed with caution, although relative mean changes in Modelflow-derived values seem to be reliable (Harms et al. 1999).

In conclusion, blood volume and sub tolerance cardiovascular responses were better maintained after bed rest in women who performed aerobic and resistive exercise countermeasures. Interestingly, the improvements did not result in a significantly longer orthostatic tolerance time.

Acknowledgments We thank the outstanding 24 women who volunteered for this bed rest investigation. We thank the nurses, staff, and entire research team at the MEDES Space Clinic (Toulouse Rangueil Hospital) for their exceptional care of the subjects during bed rest and exercise. Special thanks to Drs M-P. Bareille and A. Beck for coordination and medical monitoring, D. Greaves for technical assistance, E. Wodey for the statistical help, and R. Tullet, S. Meuche, Alex Kowanz, Bjorn Redlich, Connie Fischer, Marius Dettmer, and Nicolas Sinanan for assistance with exercise. We also thank Dr Scott Trappe and his team for providing the flywheel resistive exercise to our subjects. The study WISE-2005 was sponsored by the European Space Agency (ESA), the National Aeronautics and Space Administration of the USA (NASA), the Canadian Space Agency (CSA), and the French "Centre National d'Etudes Spatiales" (CNES), which has been the "Promoteur" of the study according to French law. This work was also supported by a NASA grant NNJ04HF71G to AR Hargens, and CSA grant 9F007-046025/001/ST to RL Hughson. The study has been performed by MEDES, Institute for Space Physiology and Medicine in Toulouse, France.

Open Access This article is distributed under the terms of the Creative Commons Attribution Noncommercial License which permits any noncommercial use, distribution, and reproduction in any medium, provided the original author(s) and source are credited.

\section{References}

Alkner BA, Tesch PA (2004) Knee extensor and plantar flexor muscle size and function following 90 days of bed rest with or without resistance exercise. Eur J Appl Physiol 93:294-305. doi:10.1007/ s00421-004-1172-8

Arbeille P, Pavy-le Traon A, Fomina G, Vasseur P, Guell A (1995) Femoral flow response to lower body negative pressure: an orthostatic tolerance test. Aviat Space Environ Med 66:131-136

Arbeille P, Kerbeci P, Mattar L, Shoemaker JK, Hughson RL (2008) WISE-2005: tibial and gastrocnemius vein and calf tissue response to LBNP after a 60-day bed rest with and without countermeasures. J Appl Physiol 104:938-943. doi:10.1152/japplphysiol. 01021.2007

Belin de Chantemele E, Blanc S, Pellet N, Duvareille M, Ferretti G, Gauquelin-Koch G, Gharib C, Custaud MA (2004a) Does resistance exercise prevent body fluid changes after a 90 -day bed rest? Eur J Appl Physiol 92:555-564. doi:10.1007/s00421-004-1121-6

Belin de Chantemele E, Pascaud L, Custaud MA, Capri A, Louisy F, Ferretti G, Gharib C, Arbeille P (2004b) Calf venous volume during stand-test after a 90-day bed-rest study with or without exercise countermeasure. J Physiol 561:611-622. doi:10.1113/ jphysiol.2004.069468

Belin de Chantemele E, Gauquelin-Koch G, Duvareille M, Pellet N, Gharib C, Custaud MA (2006) Blood volume measurement: the comparison of pulse dye densitometry and Dill and Costill's methods. Life Sci 78:1564-1569. doi:10.1016/j.lfs.2005.07.025

Boda WL, Watenpaugh DE, Ballard RE, Hargens AR (2000) Supine lower body negative pressure exercise simulates metabolic and kinetic features of upright exercise. J Appl Physiol 89:649-654

Buckey JC Jr, Lane LD, Levine BD, Watenpaugh DE, Wright SJ, Moore WE, Gaffney FA, Blomqvist CG (1996) Orthostatic intolerance after spaceflight. J Appl Physiol 81:7-18

Burge CM, Skinner SL (1995) Determination of haemoglobin mass and blood volume with CO: evaluation and application of a method. J Appl Physiol 79:623-631

Butler GC, Xing HC, Northey DR, Hughson RL (1991) Reduced orthostatic tolerance following $4 \mathrm{~h}$ head-down tilt. Eur J Appl Physiol Occup Physiol 62:26-30. doi:10.1007/BF00635629

Convertino VA (1998) Gender differences in autonomic functions associated with blood pressure regulation. Am J Physiol 275:R1909-R1920

Convertino VA, Doerr DF, Stein SL (1989) Changes in size and compliance of the calf after 30 days of simulated microgravity. J Appl Physiol 66:1509-1512

Custaud MA, de Souza Neto EP, Abry P, Flandrin P, Millet C, Duvareille M, Fortrat JO, Gharib C (2002) Orthostatic tolerance and spontaneous baroreflex sensitivity in men versus women after 7 days of head-down bed rest. Auton Neurosci 100:66-76. doi:10.1016/S1566-0702(02)00132-7 
Demiot C, Dignat-George F, Fortrat JO, Sabatier F, Gharib C, Larina I, Gauquelin-Koch G, Hughson R, Custaud MA (2007) WISE 2005: chronic bed rest impairs microcirculatory endothelium in women. Am J Physiol Heart Circ Physiol 293:H3159-H3164. doi:10.1152/ajpheart.00591.2007

Dorfman TA, Levine BD, Tillery T, Peshock RM, Hastings JL, Schneider SM, Macias BR, Biolo G, Hargens AR (2007) Cardiac atrophy in women following bed rest. J Appl Physiol 103:8-16. doi:10.1152/japplphysiol.01162.2006

Edgell H, Zuj KA, Greaves DK, Shoemaker JK, Custaud MA, Kerbeci P, Arbeille P, Hughson RL (2007) WISE-2005: adrenergic responses of women following 56-days, 6 degrees head-down bed rest with or without exercise countermeasures. Am J Physiol Regul Integr Comp Physiol 293:R2343-R2352

el-Bedawi KM, Hainsworth R (1994) Combined head-up tilt and lower body suction: a test of orthostatic tolerance. Clin Auton Res 4:41-47

Fischer D, Arbeille P, Shoemaker JK, O'Leary DD, Hughson RL (2007) Altered hormonal regulation and blood flow distribution with cardiovascular deconditioning after short-duration head down bed rest. J Appl Physiol 103:2018-2025. doi:10.1152/ japplphysiol.00121.2007

Fortney SM, Beckett WS, Carpenter AJ, Davis J, Drew H, LaFrance ND, Rock JA, Tankersley CG, Vroman NB (1988) Changes in plasma volume during bed rest: effects of menstrual cycle and estrogen administration. J Appl Physiol 65:525-533

Fortney SM, Turner C, Steinmann L, Driscoll T, Alfrey C (1994) Blood volume responses of men and women to bed rest. J Clin Pharmacol 34:434-439

Fu Q, Arbab-Zadeh A, Perhonen MA, Zhang R, Zuckerman JH, Levine BD (2004) Hemodynamics of orthostatic intolerance: implications for gender differences. Am J Physiol Heart Circ Physiol 286:H449-H457. doi:10.1152/ajpheart.00735.2002

Guell A, Braak L, Le Traon AP, Gharib C (1991) Cardiovascular adaptation during simulated microgravity: lower body negative pressure to counter orthostatic hypotension. Aviat Space Environ Med 62:331-335

Hargens AR, Whalen RT, Watenpaugh DE, Schwandt DF, Krock LP (1991) Lower body negative pressure to provide load bearing in space. Aviat Space Environ Med 62:934-937

Harm DL, Jennings RT, Meck JV, Powell MR, Putcha L, Sams CP, Schneider SM, Shackelford LC, Smith SM, Whitson PA (2001) Invited review: gender issues related to spaceflight: a NASA perspective. J Appl Physiol 91:2374-2383

Harms MP, Wesseling KH, Pott F, Jenstrup M, Van Goudoever J, Secher NH, Van Lieshout JJ (1999) Continuous stroke volume monitoring by modelling flow from non-invasive measurement of arterial pressure in humans under orthostatic stress. Clin Sci Lond 97:291-301. doi:10.1042/CS19990061

Khan MH, Kunselman AR, Leuenberger UA, Davidson WR Jr, Ray CA, Gray KS, Hogeman CS, Sinoway LI (2002) Attenuated sympathetic nerve responses after 24 hours of bed rest. Am J Physiol Heart Circ Physiol 282:H2210-H2215

Kimmerly DS, Wong SW, Salzer D, Menon R, Shoemaker JK (2007) Forebrain regions associated with postexercise differences in autonomic and cardiovascular function during baroreceptor unloading. Am J Physiol 293:H299-H306

Lee SM, Moore AD Jr, Fritsch-Yelle JM, Greenisen MC, Schneider SM (1999) Inflight exercise affects stand test responses after space flight. Med Sci Sports Exerc 31:1755-1762. doi:10.1097/ 00005768-199912000-00010
Levine BD, Zuckerman JH, Pawelczyk JA (1997) Cardiac atrophy after bed-rest deconditioning: a nonneural mechanism for orthostatic intolerance. Circulation 96:517-525

Lightfoot JT, Febles S, Fortney SM (1989) Adaptation to repeated presyncopal lower body negative pressure exposures. Aviat Space Environ Med 60:17-22

Maillet A, Zaouali-Ajina M, Vorobiev D, Blanc S, Pastouchkova L, Reushkina G, Morukov B, Grigoriev AI, Gharib C, GauquelinKoch G (2000) Orthostatic tolerance and hormonal changes in women during 120 days of head-down bed rest. Aviat Space Environ Med 71:706-714

Meck JV, Reyes CJ, Perez SA, Goldberger AL, Ziegler MG (2001) Marked exacerbation of orthostatic intolerance after long- vs. short-duration spaceflight in veteran astronauts. Psychosom Med 63:865-873

Murthy G, Watenpaugh DE, Ballard RE, Hargens AR (1994) Exercise against lower body negative pressure as a countermeasure for cardiovascular and musculoskeletal deconditioning. Acta Astronaut 33:89-96. doi:10.1016/0094-5765(94)90112-0

Pavy-Le Traon A, Louisy F, Vasseur-Clausen P, Guell A, Gharib C (1999) Contributory factors to orthostatic intolerance after simulated weightlessness. Clin Physiol 19:360-368. doi:10.1046/ j.1365-2281.1999.00189.x

Pitt MS, Marshall P, Diesch JP, Hainsworth R (2004) Cardiac output by Portapres. Clin Sci Lond 106:407-412. doi:10.1042/ CS20030279

Rittweger J, Frost HM, Schiessl H, Ohshima H, Alkner B, Tesch P, Felsenberg D (2005) Muscle atrophy and bone loss after 90 days' bed rest and the effects of flywheel resistive exercise and pamidronate: results from the LTBR study. Bone 36:1019-1029. doi:10.1016/j.bone.2004.11.014

Schneider SM, Watenpaugh DE, Lee SM, Ertl AC, Williams WJ, Ballard RE, Hargens AR (2002) Lower-body negative-pressure exercise and bed-rest-mediated orthostatic intolerance. Med Sci Sports Exerc 34:1446-1453. doi:10.1097/00005768-20020900000008

Sigaudo-Roussel D, Custaud MA, Maillet A, Guell A, Kaspranski R, Hughson RL, Gharib C, Fortrat JO (2002) Heart rate variability after prolonged spaceflights. Eur J Appl Physiol 86:258-265. doi:10.1007/s00421-001-0551-7

Traon AP, Vasseur P, Arbeille P, Guell A, Bes A, Gharib C (1995) Effects of 28-day head-down tilt with and without countermeasures on lower body negative pressure responses. Aviat Space Environ Med 66:982-991

Vernikos J, Dallman MF, Keil LC, O’Hara D, Convertino VA (1993) Gender differences in endocrine responses to posture and 7 days of -6 degrees head-down bed rest. Am J Physiol 265:E153-E161

Watenpaugh DE, Ballard RE, Schneider SM, Lee SM, Ertl AC, William JM, Boda WL, Hutchinson KJ, Hargens AR (2000) Supine lower body negative pressure exercise during bed rest maintains upright exercise capacity. J Appl Physiol 89:218-227

Watenpaugh DE, O'Leary DD, Schneider SM, Lee SM, Macias BR, Tanaka K, Hughson RL, Hargens AR (2007) Lower body negative pressure exercise plus brief postexercise lower body negative pressure improve post-bed rest orthostatic tolerance. J Appl Physiol 103:1964-1972. doi:10.1152/japplphysiol.00132.2007

Wesseling KH, Jansen JR, Settels JJ, Schreuder JJ (1993) Computation of aortic flow from pressure in humans using a nonlinear, threeelement model. J Appl Physiol 74:2566-2573 